\title{
Panniculitis-induced localized lipodystrophy
}

INSERM

\section{Source}

INSERM. (1999). Orphanet: an online rare disease and orphan drug data base.

Panniculitis-induced localized lipodystrophy. ORPHA:90159

Panniculitis-induced localized lipodystrophy is a rare, acquired, localized lipodystrophy disorder characterized by eruption of tender, occasionally painful, erythematous nodules and plaques which enlarge radially and resolve into lipoatrophic lesions, often located in the upper and lower limbs. Histologically, lesions are characterized by lipophagic, lobular panniculitis and absence of vasculitis. 